\title{
Severe psoriasis emerged after treatment of chronic HCV co-infection with pegylated- interferon and ribavirin, supported by neutropenia rescue with repeated filgrastim administration
}

\author{
Roberto Manfredi', Sergio Sabbatani \\ From $16^{\text {th }}$ International Symposium on HIV and Emerging Infectious Diseases \\ Marseille, France. 24-26 March 2010
}

\section{Background}

Associated treatment with pegylated interferon plus specific antiviral compounds significantly improved the prognosis of chronic hepatitis $C$ and hepatitis $B$ even when an underlying HIV infection is of concern, although antiviral drugs (especially interferon and its derivatives), tend to be myelotoxic and also some rescue treatments, like human recombinant granulocyte colony-stimulating factors (G-CSF, filgrastim or lenograstim) (which are extensively administered in order to correct neutropenia induced by antiviral therapy), may also be involved in prompting or exacerbating cutaneous psoriasis and its systemic complications.

\section{Methods}

A representative case report of a $\mathrm{HCV}$-monoinfected woman with no personal and familial history of psoriasis, who suffered from a chronic, progressive, evolutive hepatitis $C$, underwent long-term treatment with combined pegylated interferon plus ribavirin, and resorted to multiple cycles of G-CSF (filgrastim), in order to recover a severe, recurring granuloytopenia caused by antiviral therapy itself, and to maintain an effective dosage of both anti-HCV antivirals.

\section{Results}

Five months after treatment initiation, she suddenly developed an extensive and severe cutaneous psoriasis, which did not benefit from all attempted local therapies, and improved

* Correspondence: Roberto.manfredi@unibo.it

Infectious Diseases, University of Bolognna, S. Orsola Hospital, Bologna, Italy only after specific cyclosporin treatment. Anti-HCV therapy was first interrupted, and then continued with a lowered dosages of peg-interferon and ribavirin, so that a sustained anto-HCV therapeutic response was not obtained.

\section{Discussion}

From a pathogenetic point of view, in our case it remains extremely difficult to distinguish the role of pergylated interferon from that of the accompanying ribavirin, from that of the frequently administered granulocyte growth factor (filgrastim in our case), since all mentioned drugs were administered concurrently during many months, and according to the existing literature evidences, all of them have a potential to induce/exacerbate psoriasis as a potential untoward effect in subjects suffering from chronic hepatitis. Cyclosporin treatment obtained a stable remission of this last severe cutaneous complication, but the efforts to contain the progression the underlying evolutive hepatitis $\mathrm{C}$ were blunted by the difficult-to-treat genotype $1 \mathrm{HCV}$ infection, and the frequent need to lower drug dosages and/or to interrupt antiviral therapy, because of initial myelotoxicity and subsequent cutaneous complications, probably driven by anti-HCV therapy itself.

Published: 11 May 2010

doi:10.1186/1742-4690-7-S1-P84

Cite this article as: Manfredi and Sabbatani: Severe psoriasis emerged after treatment of chronic HCV co-infection with pegylated-interferon and ribavirin, supported by neutropenia rescue with repeated filgrastim administration. Retrovirology 2010 7(Suppl 1):P84. 